\title{
Flashboiling atomization in nozzles for GDI engines
}

\author{
Sebastian Bornschlegel $^{\star 12}$, Chris Conrad ${ }^{12}$, Lisa Eichhorn ${ }^{12}$, Michael Wensing ${ }^{12}$ \\ ${ }^{1}$ Institute of Engineering Thermodynamics (LTT), FAU Erlangen-Nürnberg, Germany \\ ${ }^{2}$ Erlangen Graduate School in Advanced Optical Technologies (SAOT), \\ FAU Erlangen-Nürnberg, Germany \\ *Corresponding author: sebastian.bornschlegel@fau.de
}

\begin{abstract}
Flashboiling denotes the phenomenon of rapid evaporation and atomization at nozzles, which occurs when fluids are injected into ambient pressure below their own vapor pressure. It happens in gasoline direct injection (GDI) engines at low loads, when the cylinder pressure is low during injection due to the closed throttle valve. The fuel temperature at the same time approaches cylinder head coolant temperature due to the longer dwell time of the fuel inside the injector. Flash boiling is mainly beneficial for atomization quality, since it produces small droplet sizes and relative broad and homogenous droplet distributions within the spray. Coherently, the penetration depth normally decreases due to the increased aerodynamic drag. Therefore the thermal properties of injectors are often designed to reach flash boiling conditions as early as possible. At the same time, flash boiling significantly increases the risk of undesired spray collapsing. In this case, neighbouring jets converge and form a single jet. Due to the now concentrated mass, penetration depth is enhanced again and can lead to liner or piston wetting in addition to the overall diminished mixture formation.

In order to understand the underlying physics, it is important to study the occurring phenomena flashboiling and jet-to-jet interacting i.e. spray collapsing separately. To this end, single hole injectors are built up to allow for an isolated investigation of flashboiling. The rapid expansion at the nozzle outlet is visualized with a microscopic high speed setup and the forces that lead to the characteristic spray expansion are discussed. Moreover, the results on the macroscopic spray in terms of penetration, cone angles and vapor phase are shown with a high speed Schlieren setup. Resulting droplet diameters and velocities are measured using LDA/PDA.

As a result, we find a comprehensive picture of flash boiling. The underlying physics can be described and discussed for the specific case of high pressure injection at engine relevant nozzle geometries and conditions, but independently from neighbouring jets. These findings provide the basis to understand and investigate flashboiling and jet-to-jet interaction as distinct, but interacting subjects rather than a combined phenomenon.
\end{abstract}

\section{Keywords}

Flashboiling, GDI, Single-Hole-Injector, High-Speed, LDA/PDA

\section{Introduction}

With the introduction of gasoline direct injection (GDI) systems in significant shares in the worldwide market, new challenges for spray and combustion layout were generated, originating from the increased injection pressure as well as the hotter injector and fuel temperatures due to the changed injector mounting position.

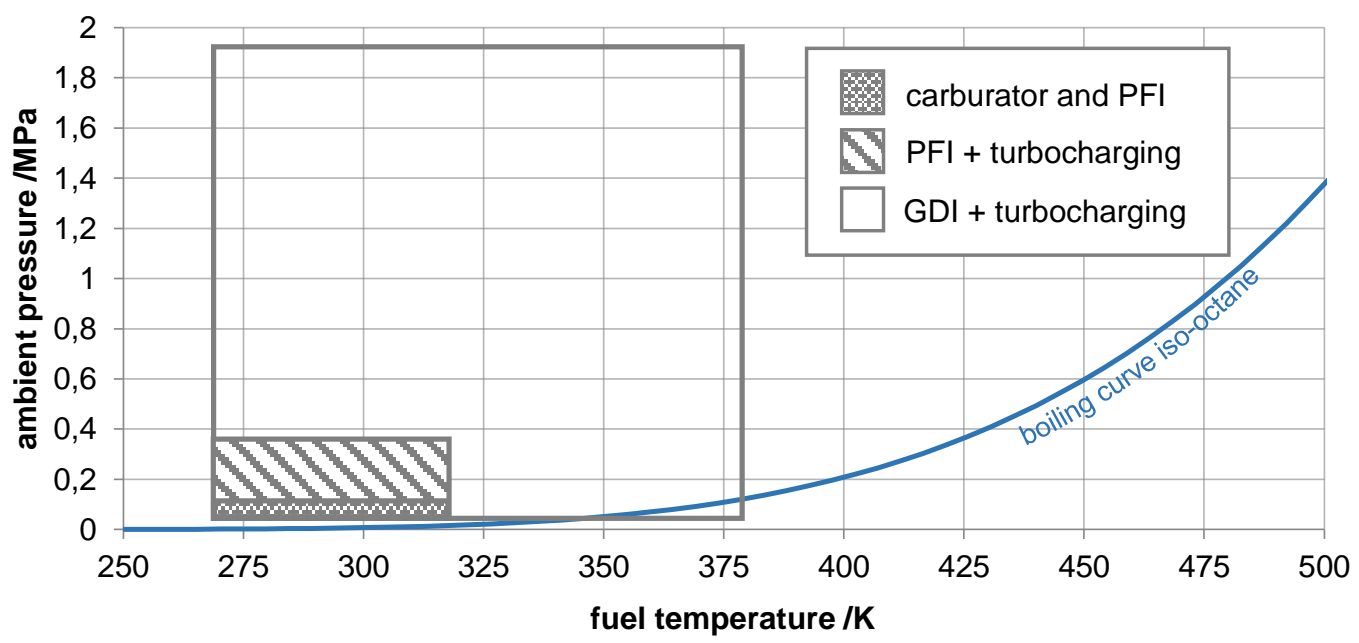

Figure 1. p-T diagram of iso-octane with possible fuel conditions for different injection systems 
Figure 1 shows estimated possible injection conditions relative to the boiling curve of the fuel surrogate iso-octane for different injection systems, particularly. One effect that is in focus ever since GDI entered the market is flashboiling. It denotes the phenomenon of rapid evaporation and atomization at nozzles, which occurs when fluids are injected into ambient pressure below their own vapor pressure and correlates to the lower right corner in Figure 1. In GDI engines, this happens at low loads, when the cylinder pressure is low due to closed throttle valves. At the same time, the dwell time of the fuel inside the injector increases due to low injected mass and low engine revolutions, causing the fuel to approach cylinder head coolant temperature. During injection the pressure then drops below the fuels vapor pressure as indicated in Figure 2 for an exemplary fuel pressure of $15 \mathrm{MPa}$ and the fuel-surrogate iso-octane, where the vertical arrow displays an isenthalpic throttling from in-injector conditions to chamber conditions. The horizontal arrows mark the separation of liquid and vapor phase due to superheating, with the respective arrow length representing vapor and liquid fractions. Real gasoline fuel in contrast to iso-octan is a multi-component mixture. This means that the boiling curve is no well-defined line but the mixture evaporates in a certain range instead. This means for real fuel, a small fraction of the mixture is superheated under nearly all conditions. Iso-octane however is a good average for the components in real fuel and shows comparable behaviour.

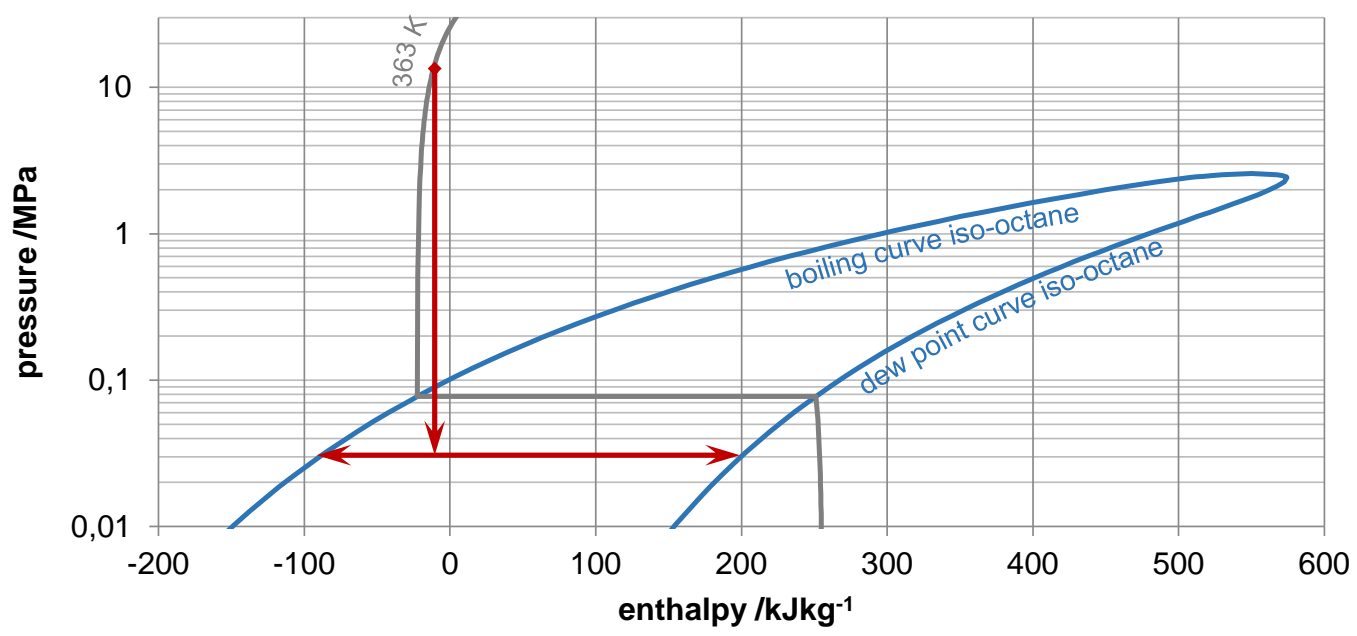

Figure 2. p-h-diagramm of iso-octane with an exemplary injection from $15 \mathrm{MPa}$ fuel pressure to $0.03 \mathrm{MPa}$ ambient pressure at $363 \mathrm{~K}$ fuel temperature

Flashboiling is usually described as a three step process [1],[2]. When the degree of superheating is so high that enthalpy cannot be emitted by heat transfer and evaporation at the free surface, evaporation starts within the fluid volume, causing the formation of gas bubbles. This effect is referred to as nucleation. Those nuclei grow during the next step which is referred to as bubble growth, till they reach an instable size. In the last state of flashboiling they collapse, which leads to the creation of new free surface and consequently boosts atomization [3]. Wood shows a decrease in the Sauter-Mean diameter $D_{32}$ of $42 \%$ for a flash boiling spray [4]. The spray used however is a six-hole spray, that shows considerable collapsing under flash boiling conditions. In this case, neighbouring jets converge and form a single jet. Due to the now concentrated mass, penetration depth is enhanced again and can lead to liner or piston wetting in addition to the overall diminished mixture formation. Zeng shows this behaviour for an eight-hole injector [5]. For low degrees of superheating, spray width is enhanced and penetration depth decreased. For higher degrees of superheating the spray collapses and the spray penetration increases again while the spray width decreases. Krämer points out that flashboiling and jet-to-jet interaction are separate phenomena that however influence each other [6]. In order to understand the underlying physics of both effects, it is important to study the occurring phenomena flashboiling and jet-to-jet interacting i.e. spray collapsing separately. To this end, in this paper single hole injectors are used to allow for an isolated investigation of flashboiling.

\section{Material and methods}

The injector used is a common GDI solenoid injector which however has just one single hole. To keep the inflow conditions as realistic as possible, the hole is not placed centrally but on a usual reference circle, resulting in a jet height-angle of $30^{\circ}$ from injector axis. Both hole diameter and L/D ratio are unchanged from the original multi-hole design. The injectors are investigated with three different measurement techniques and results from four 
operation points (OP) are shown in this paper as depicted in Table 1. Each OP is defined by fuel pressure, fuel temperature, chamber pressure, chamber temperature and the injection duration $t_{i}$.

Table 1. Operation Points

\begin{tabular}{|c|c|c|c|c|c|c|c|}
\hline OP & $\bar{T}_{\text {fuel }}$ & $p_{\text {fuel }}$ & $\overline{T_{\text {chamber }}}$ & $\mathbf{p}_{\text {chamber }}$ & $\mathbf{t}_{\mathbf{i}}$ & $\mathrm{DS}_{\mathrm{p}}$ & $\mathrm{DS}_{\mathrm{h}}$ \\
\hline 1 & \multirow{2}{*}{$303 \mathrm{~K}$} & \multirow{4}{*}{$15 \mathrm{MPa}$} & \multirow{4}{*}{$298 \mathrm{~K}$} & $0.1 \mathrm{MPa}$ & \multirow{4}{*}{$1.5 \mathrm{~ms}$} & 0,08 & -0,53 \\
\hline 2 & & & & $0.03 \mathrm{MPa}$ & & 0,28 & $-0,19$ \\
\hline 3 & \multirow{2}{*}{$363 \mathrm{~K}$} & & & $0.1 \mathrm{MPa}$ & & 0,77 & $-0,03$ \\
\hline 4 & & & & $0.03 \mathrm{MPa}$ & & 2,58 & 0,27 \\
\hline
\end{tabular}

All measurements are performed with iso-octane as a fuel surrogate, due to its well-known properties. The degree of superheating generally can be represented by two expressions, the ratio of vapor pressure to ambient pressure as shown in equation (1) or the ratio of inherent enthalpy of the fluid to the enthalpy of phase transition as shown in (2). The latter is partly similar to the dimensionless Jacob-number that however often is defined in a volumespecific way [7].

$$
\begin{aligned}
& D S_{p}=\frac{p_{\text {sat }}\left(T_{i n j}\right)}{p_{a}} \\
& D S_{h}=\frac{h_{i n j}-h_{l}}{h_{v}-h_{l}}
\end{aligned}
$$

Index "sat" refers to saturation conditions at the state point on the boiling curve defined by the thermodynamic quantity given in brackets. Index "inj" refers to the conditions inside the injector, while index "a" refers to ambient i.e. chamber conditions. $\mathrm{h}$ is the enthalpy and indices "l" and " $\mathrm{v}$ " refer to the liquid and vapor phase. While the first definition is common in technical applications, because the necessary values are gained more easily also for real multi-component fuels, the enthalpy-based definition provides more information. A negative value means the fluid is not superheated while values between 0 and 1 give the amount of fuel that can be evaporated solely by the inherent enthalpy of the fuel, and a value above 1 means that full phase transition can be achieved during flashboiling. The values from both definitions are given in Table 1. In this case, only OP 4 provides real superheated conditions, while OP 2 and 3 show the effects of reducing chamber pressure and increasing fuel temperature respectively, without crossing the boiling curve of iso-octane.

Three measurement techniques are used to examine the spray. The full spray behaviour is visualized by high speed Schlieren imaging. To this end a folded Z-type Schlieren setup with 6 inch parabolic field mirrors is used. The light source is a high-power LED array in cw-mode, the camera a Photron SA-Z high-speed-camera that is used at a repetition rate of $20 \mathrm{kHz}$. The images are used to determine macroscopic spray paramters e.g. penetration depth and cone angle. Due to its sensitivity towards gradients in the refractive index, the Schlieren measurement technique is sensitive towards both liquid and vapor phase. To verify the obtained results, they were compared to results from two different integral spray measurement techniques, Mie scattering and shadowgraphy. It can be shown that for the operation points considered in this publication, the Schlieren measurement technique shows the same results as the other two techniques that are only sensitive towards the liquid phase due to their measurement principle. This means that a separation of liquid and vapor phase does not occur in the spray on a macroscopic scale and that the results are therefore not depending on the sensitivity of the measurement technique towards gaseous fuel. This however is only true for operation points with comparably slow heat transfer from the surrounding gas phase and therefore low evaporation rates.

To provide microscopic spray parameters i.e. droplet sizes and velocities, a commercial Dantec fibre PDA system is used. In total 119 measurement positions are investigated for each operation point, with a repeat count of 60 injections for each position. All measurement positions lie in a common plane through both injector and jet axis as depicted in Figure 6.

Finally the nozzle near region is investigated by line of sight close up measurements in ultra-high-speed with framerates of 5 and 2 million frames per second to investigate the initial process of flashboiling itself. To this end a SI-AD 500 high power flash lamp and a Kirana camera were used, both provided by Specialised Imaging, in combination with a Questar QM-100 long distance microscope as well as a Navitar 12x Zoom Lense.

\section{The effect of flashboiling on macroscopic spray parameters}

A jet usually is described by two basic macroscopic parameters, its penetration depth and its jet cone angle. For the latter, a large number of definitions are in use, depending of the particular application. In this paper, a 
definition proposed in [8] is used. In addition, the jet volume is calculated under the assumption of an axissymmetrical spray-shape. To give a visual impression of the effect of flashboiling on the spray shape, in Figure 3 raw images for each OP are compared at a similar time after actuating of the injector i.e. after electrical start of injection (eSOI).
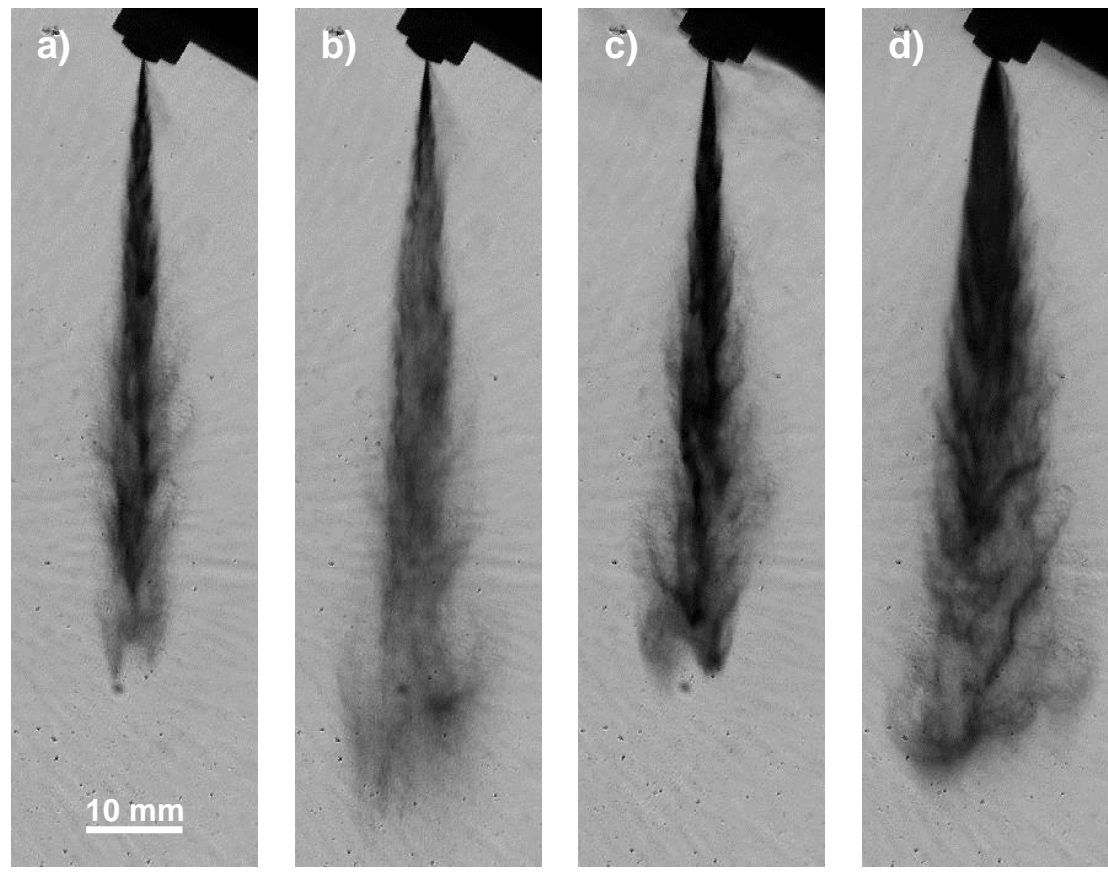

Figure 3. Raw Schlieren images $1000 \mu \mathrm{s}$ after eSOI for a) OP1 (303 K / $0.1 \mathrm{MPa})$, b) OP2 (303 K / $0.03 \mathrm{MPa})$, c) OP3 (363 K/ $0.1 \mathrm{MPa})$ and d) OP4 (363 K / $0.03 \mathrm{MPa})$

Even though injection pressure and duration are kept constant, the spray form changes significantly between the four OPs. On the upper side of the spray, for each OP a small fog of droplets is visible, that is most likely caused by the changed inflow of the single hole nozzle. This however can be neglected in evaluation and the jet itself behaves comparable to a jet from a multi hole injector. The different spray forms are also visible in the temporal evaluation of the high speed images.
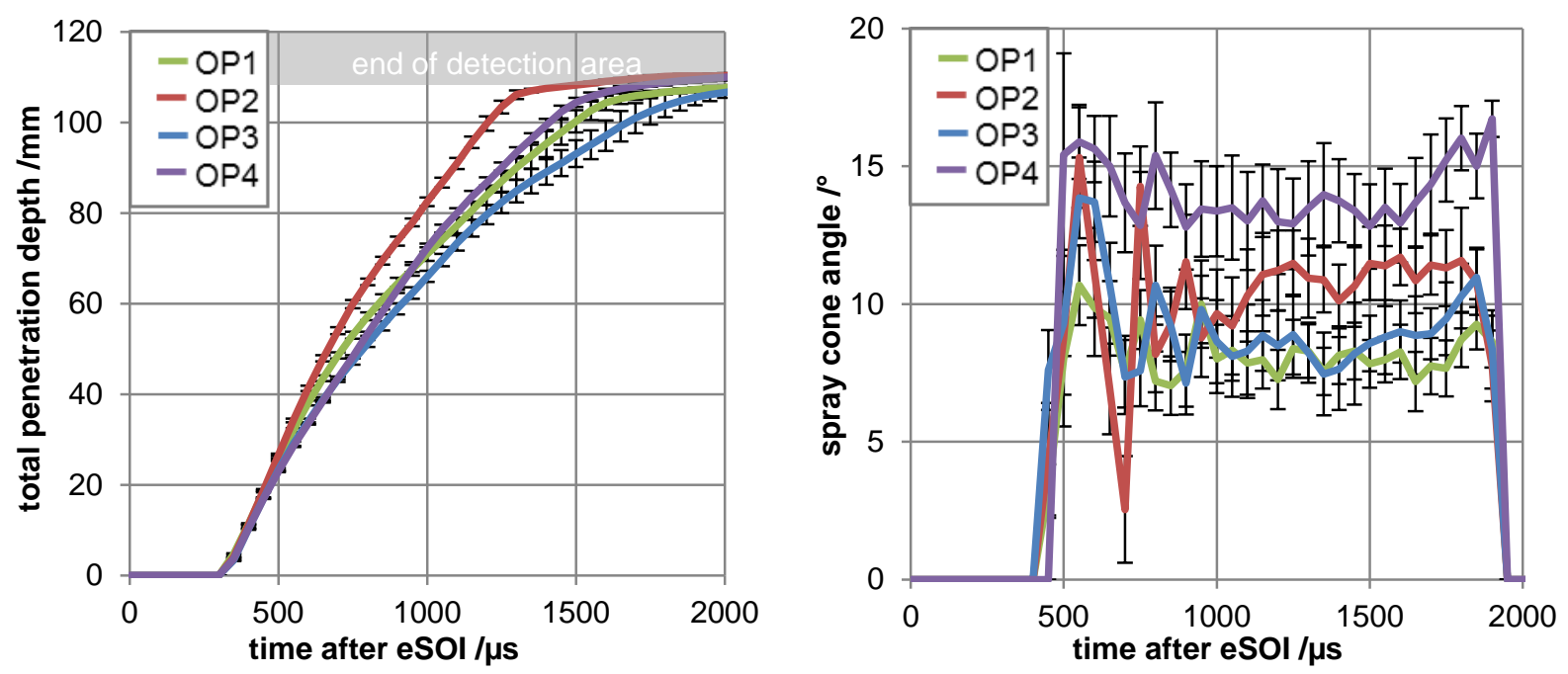

Figure 4. Total penetration depth and spray cone angle relative to time after eSOI for OP1 ( $303 \mathrm{~K} / 0.1 \mathrm{MPa}), \mathrm{OP} 2(303 \mathrm{~K} / 0.03$ $\mathrm{MPa})$, OP3 (363 K / $0.1 \mathrm{MPa})$ and OP4 (363 K/ $0.03 \mathrm{MPa})$

Figure 4 on the left shows the temporal development of the total spray penetration depth (absolute distance between spray front and nozzle outlet). Note that at around $105 \mathrm{~mm}$ the end of the optical access and therefore 
the maximum possible penetration depth is reached. Generally an increase in penetration depth can be found for OPs with lower ambient density and therefore lower gas density. Higher fuel temperature in contrast reduces the penetration depth. Both effects are found for the non-superheated OPs as well as for the superheated one, even though the effect of heated fuel is stronger between OP2 and OP4 than between OP1 and OP3.

Figure 4 on the right shows the spray cone angle. While both OPs at $0.1 \mathrm{MPa}$ ambient pressure show similar spay cone angles of around $8^{\circ}$ in the stationary spray phase, a decrease in ambient pressure results in a wider spray angle for both hot and cold fuel conditions. For the superheated spray however, this effect is significantly stronger with a spray cone angle of around $14^{\circ}$ in the stationary phase. This effect is also visible in Figure 3 , where the spray for OP4 is noticeable broader than the other OPs.

Since the spray penetration depth only significantly diverges in regions more than $20 \mathrm{~mm}$ away from the nozzle outlet the differences have to be caused by aerodynamic drag. While the reduction of aerodynamic drag with decreasing pressure is due to the decreasing ambient density, the increase with temperature is due to larger spray front cross section that can also be obtained in the increasing spray cone angle.
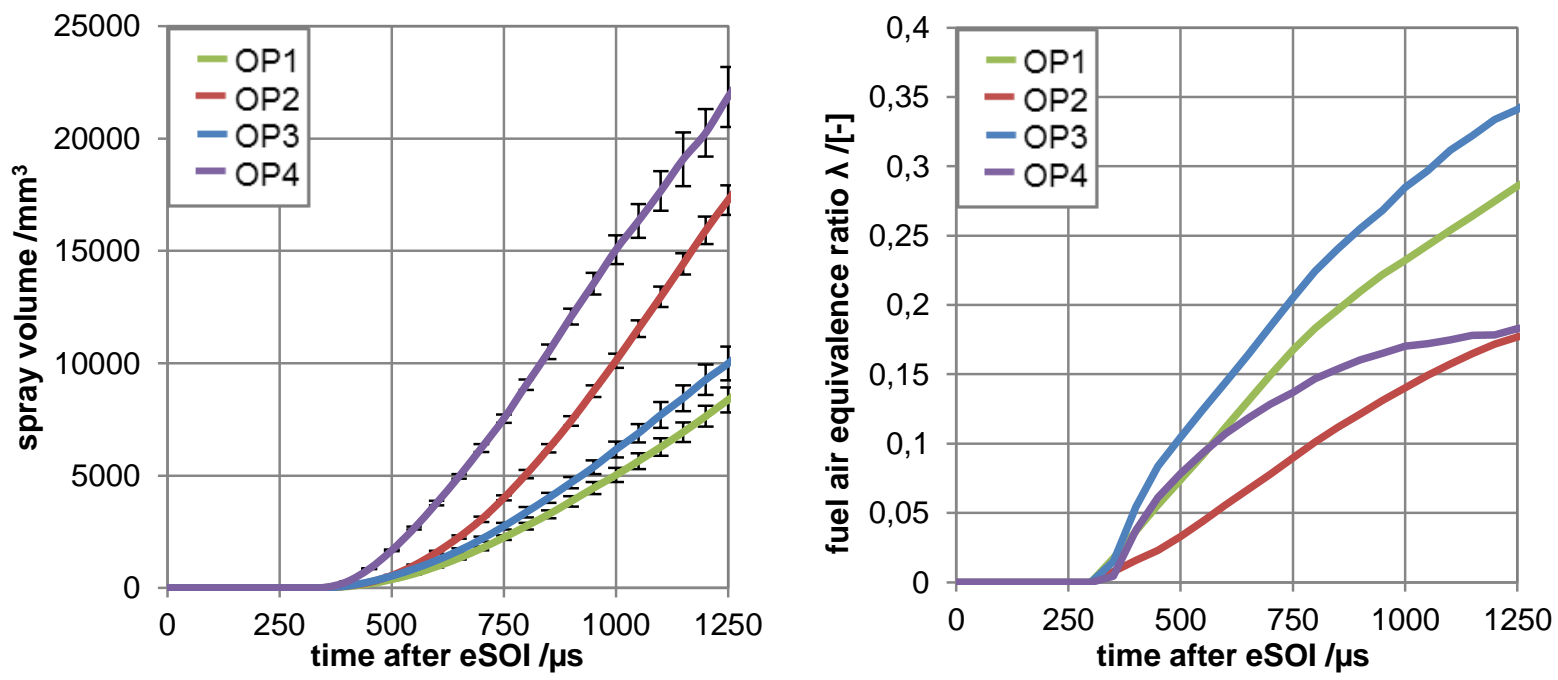

Figure 5. Spray volume and calculated fuel-air ratio relative to time after eSOI for OP1 ( $303 \mathrm{~K} / 0.1 \mathrm{MPa})$, OP2 ( $303 \mathrm{~K} / 0.03$ $\mathrm{MPa})$, OP3 (363 K / $0.1 \mathrm{MPa})$ and OP4 (363 K / $0.03 \mathrm{MPa})$

Under the assumption of an axis symmetrical jet, the spray volume can be calculated from the detected spray area. Its temporal development is given in Figure 5 on the left side. Both, an increase in fuel temperature and a decrease in ambient pressure result in an increased spray volume. While the non-superheated OPs start on the same level and diverge over time, the flash boiling spray shows a significantly increased spray volume right from the visible start of injection ( $\mathrm{vSOI}$ ).

Based on the spray volume and the injected fuel mass known from injection rate measurements, the global fuelair ratio within the spray can be estimated. This is based on the two assumption that the injected fuel mass is constant for all 4 OPs and that the liquid-vapor fraction of the fuel can be estimated assuming isenthalpe throttling. Both assumptions are simplifications, as a change in mass-flow due to changed fuel properties is neglected as well as enthalpy that adds to energy forms different than heat of evaporation, such as free surface energy. Nevertheless, an estimation of the fuel-air ratio is possible as the neglected terms are sufficiently small. The assumptions given lead to the following mathematical expression:

$$
\lambda=\frac{n_{O_{2}}}{n_{O_{2}, \text { min }}}=\frac{\frac{V_{\text {spray }}-\left(m_{\text {fuel }} \cdot \rho_{\text {fuel }}\right)}{V_{\text {mol }}}}{m_{\text {fuel }} \cdot A_{\text {st }}}
$$

The minimum stoichiometric oxygen amount $\mathrm{n}_{\mathrm{O} 2 \text {, min }}$ can be calculated with the known fuel mass $\mathrm{m}_{\text {fuel }}$ and the stoichiometric air consumption $\mathrm{A}_{\mathrm{st}}$ of iso-octane. The actual oxygen amount results from the difference between spray volume and estimated fuel volume, converted by the molar volume $\mathrm{V}_{\mathrm{mol}}$. The temporal development of the calculated fuel-air equivalence ratio is given in Figure 5 on the right. It shows that even though the flashboiling OP 4 has significantly more spray volume than the other OPs, the amount of air inside the spray is lower than for the $0.1 \mathrm{MPa}$ OPs. This effect is caused by the lower air density at $0.03 \mathrm{MPa}$. Compared to the cold fuel at 
reduced ambient pressure (OP2) however, the flashboiling OP shows a significantly increased air capture in the inertial spray phase. During late injection the curves for both OPs converge. This means that the rapid inertial expansion leads to an enhanced air capture, while air entrainment might even be weakened due to air displacement by fuel vapor.

\section{The effect of flashboiling on droplet size and velocity}

The quality of atomization can be described by the sizes of the individual spray droplets. To determine those, the spray is analysed by PDA/LDA. The investigated measurement points are visualized in Figure 6 with the measurement results for each point displayed in the corresponding schlieren image. To compare the OPs individually, the values in the nozzle distance of $29 \mathrm{~mm}$ are chosen as indicated by the green line. This distance is chosen for two reasons. The first is its relevance for the application, since compared to bore and stroke of modern DI engines this is often roughly the distance between nozzle tip and the centre of the combustion chamber. The second reason is the optical density of the spray closer to the nozzle tip. In order to minimize the influence of potential measuring gaps, $29 \mathrm{~mm}$ represents a distance with a sufficient high data rate
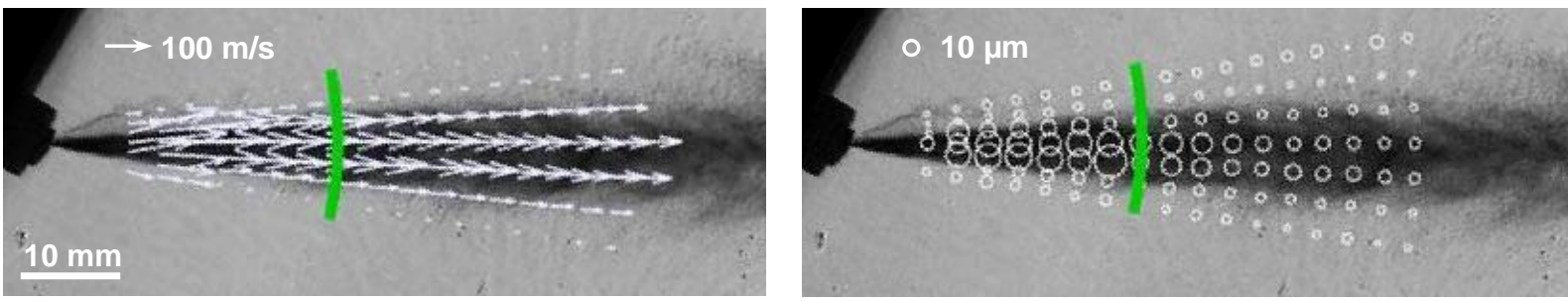

Figure 6. Mean droplet sizes (right) and velocities (left) for each of the 119 measurement points displayed in the corresponding schlieren image, all data for $1500 \mu \mathrm{s}$ after eSOI
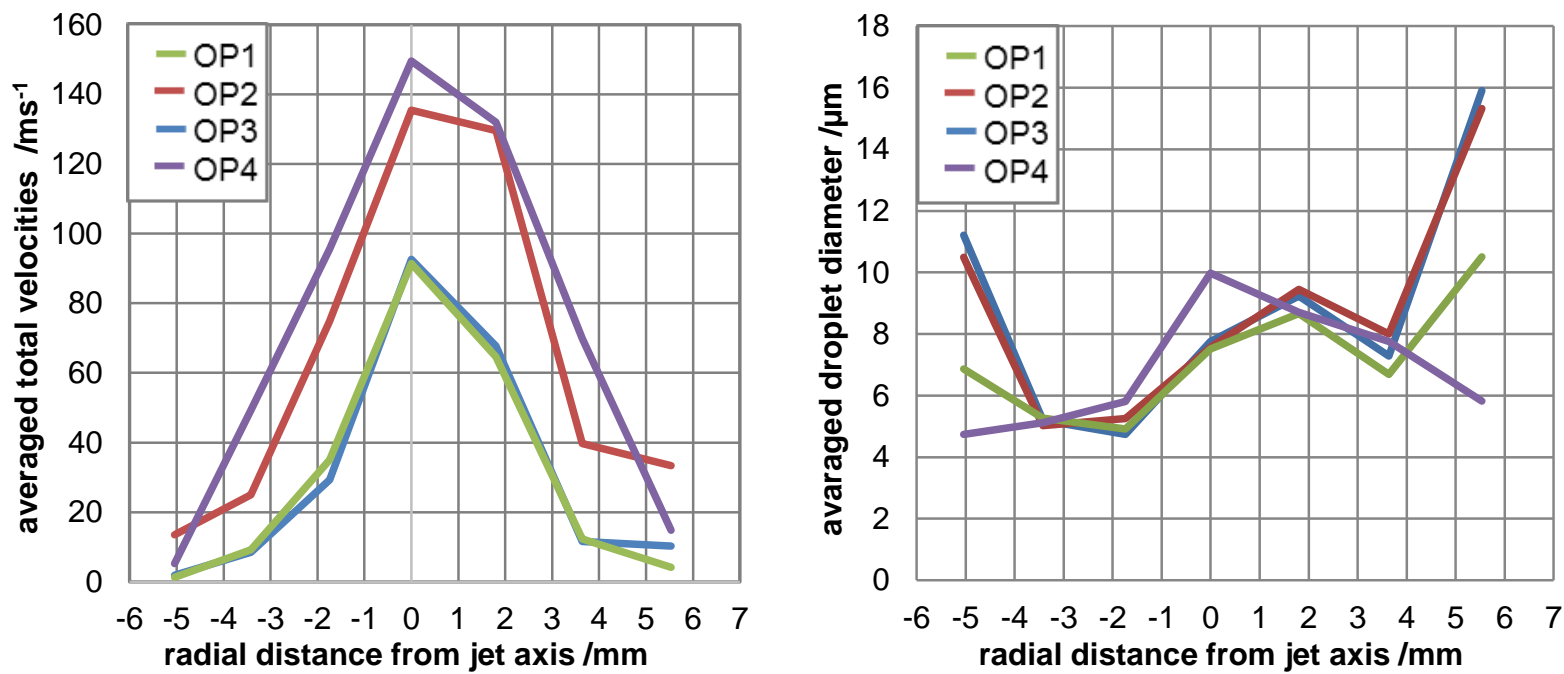

Figure 7. Radial distributions of averaged total droplet velocities (left) and droplet diamteres (right) in $29 \mathrm{~mm}$ nozzle distance for OP1 (303 K / 0.1 MPa), OP2 (303 K / $0.03 \mathrm{MPa})$, OP3 (363 K / $0.1 \mathrm{MPa})$ and OP4 (363 K / $0.03 \mathrm{MPa})$

Figure 7 shows radial droplet size and velocity distributions for the given distance. The velocities on the left show a significant dependency from the ambient pressure, while for the $0.1 \mathrm{MPa}$ OPs an influence of the fuel temperature is not detectable. For the $0.03 \mathrm{MPa}$ OPs, the superheated hot fuel OP 4 shows higher velocities than the cold fuel point. This is contrary to the macroscopic spray behaviour shown in Figure 4 . The difference is that the penetration depth is the integrated spray front velocity, while PDA measures individual droplet velocities in the spray front but also in the bulk spray phase. This means that the spray front is decelerated by increased aerodynamic drag due to the increased spray front cross section while the aerodynamic drag on droplets in the spray bulk phase is even decreased by air displacement due to the increased fuel vapor volume.

\section{Visualization of the primary atomization with and without flashboiling}

Since flashboiling is a very fast process, which takes place at the direct vicinity of the nozzle tip respectively partially inside the nozzle, a very high temporal and spatial resolution is required to visualize the process. With the 
setup described above a temporal resolution of $5 \mathrm{MHz}$ and a spatial resolution of about $4.8 \times 4 \mathrm{~mm}$ can be achieved. Since the camera is limited to 180 frames, only the opening process is analysed. For the stationary spray phase the Schlieren results show sufficient details anyway. Figure 8 shows the opening process for the reference OP 1 and the superheated OP 4 . For a clear arrangement, only every $20^{\text {th }}$ frame is shown here, which correlates to a step width of $4 \mu \mathrm{s}$ between each image, starting from vSOI. The definition of vSOI is however somewhat challenging, since for the hot fuel OPs fuel starts to leak from the injector early during needle lift due to the lower viscosity, creating a pre-jet with very little momentum. That is why in Figure 8 for the superheated OP fuel is visible outside the nozzle already at vSOI. While for the cold OP 1 spray structures and ligaments are clearly visible. For the superheated fuel only a fuel cloud is visible, which the very early stage shows very high radial expansion velocities. Only from $16 \mu \mathrm{s}$ after vSOI a contraction of the spray root and a convergence towards the later spray cone angle is visible. Even though the spray penetration depth for this OP is significantly higher than for the cold reference OP 1, as shown in Figure 9, evidence for a significant axial expansion of the spray due to flashboiling cannot be found as the comparison with the cold $0.03 \mathrm{MPa}$ OP 2 shows. This evaluation however has to be treated with care since as mentioned before, the definition of $\mathrm{vSOI}$ is challenging. An evaluation relative to eSOI on the other hand is not possible, since the camera is only able to cover a very limited timespan. In addition, the evaluation is only based on two injections per OP making shot-to-shot variation a significant source of errors.
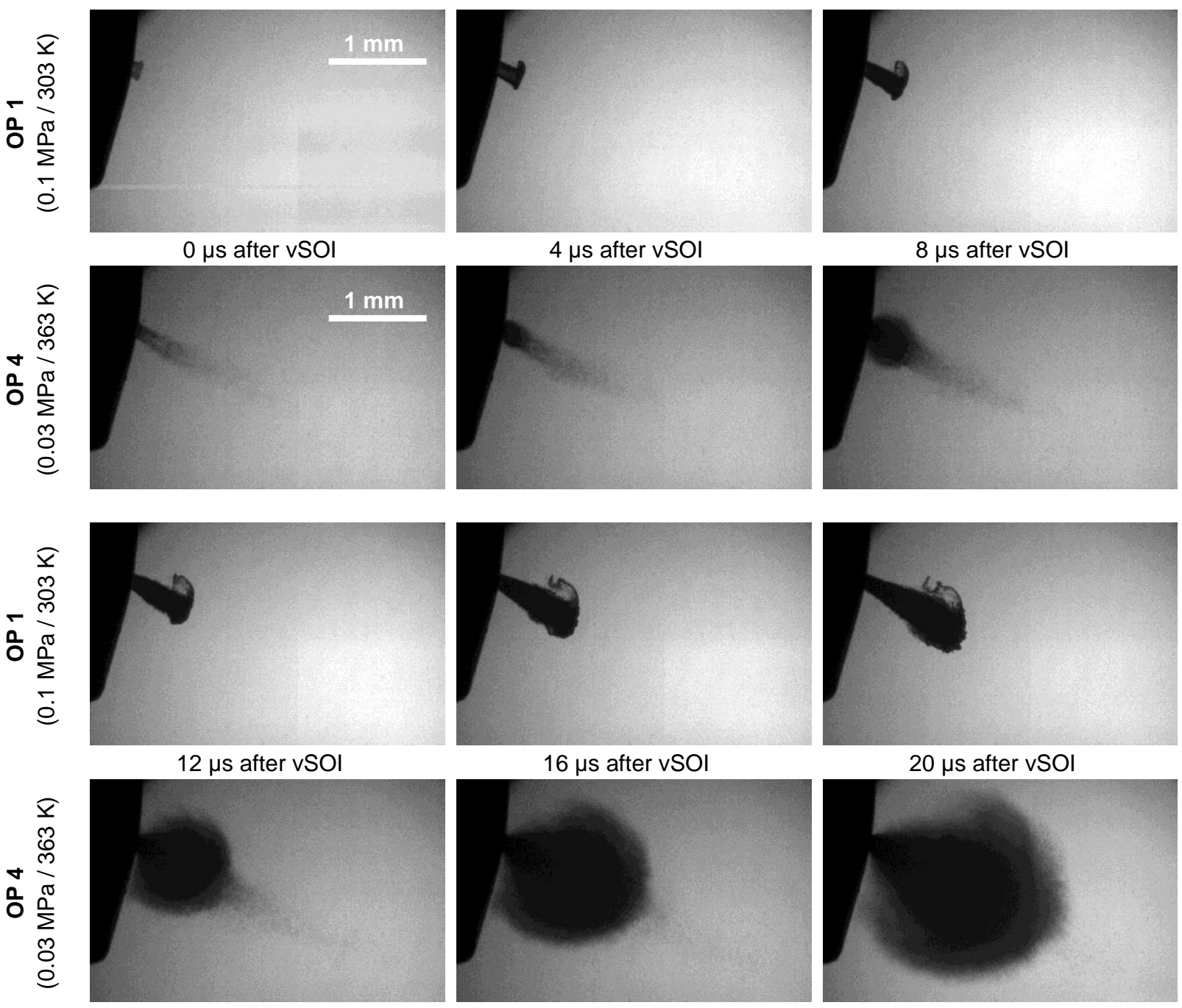

Figure 8. Visualization of the injector opening under non-superheated (OP $1-0.1 \mathrm{MPa} / 303 \mathrm{~K}-$ top row) and superheated (OP $4-0.03 \mathrm{MPa} / 303 \mathrm{~K}$ - bottom row) conditions 


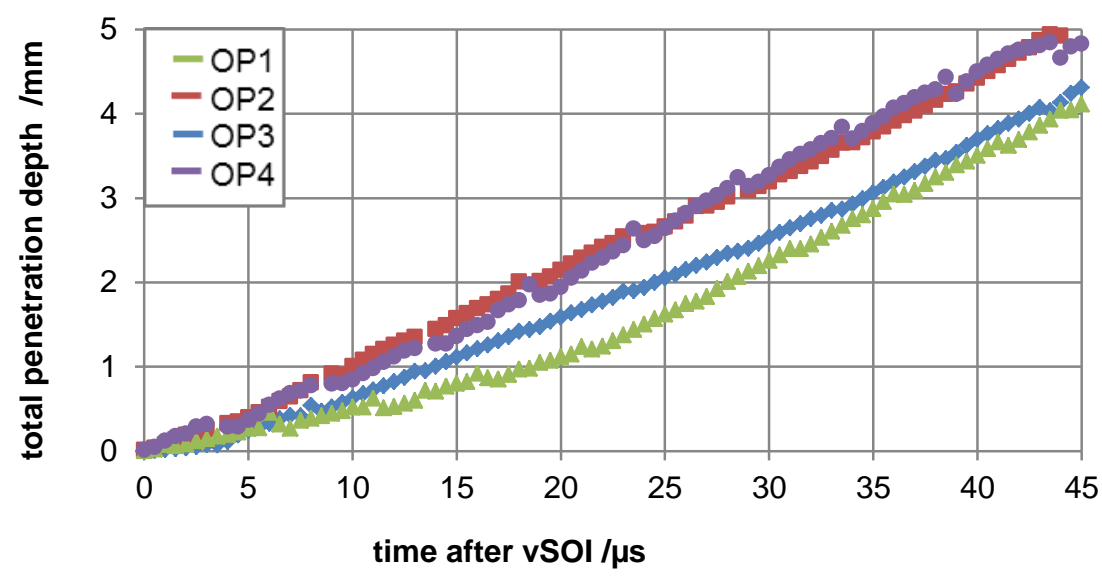

Figure 9. Total penetration depth relative to time after vSOI, obtained from close up ultra-high-speed images distance for OP1 (303 K / 0.1 MPa), OP2 (303 K / $0.03 \mathrm{MPa})$, OP3 (363 K / $0.1 \mathrm{MPa})$ and OP4 (363 K/ $0.03 \mathrm{MPa})$

\section{Conclusions}

For single hole injectors, the effects of flashboiling on the spray behavior are less significant than expected. The macroscopic spray behaviour is changed in terms of penetration depth, cone angle and spray volume, but the tendencies are already visible for the changes in fuel temperature respectively ambient pressure without superheating. Also, the enhanced primary atomization due to flashboiling has no detectable influence on the droplet size distribution in application relevant nozzle distances. This of course does not mean that flashboiling has no benefits for the GDI spray, since the evaporation of fuel is faster under those conditions, which then boosts the mixture formation in the engine.

What is noticeable in the spray however, is the behaviour in direct vicinity of the nozzle outlet where a strong radial acceleration of the spray is detectable. This leads to a significantly increased spray root width and an early increase in spray volume. The volume balance in addition hinds towards a changed and potentially extenuated air entrainment due to the significant increase of spray vapor volume. Therefore, to understand the effect of flashboiling on multi hole sprays, the interconnection to Jet-to-Jet interaction and finally spray collapsing, the flashboiling induced flow field in the surrounding air and the jet overlap in the initial spray phase, before the flow field is fully developed have to be investigated more closely.

\section{Acknowledgement}

The authors would like to thank Continental for providing the experimental injectors used in this paper and Specialised Imaging for providing parts of the equipment used for the shown measurements.

Additionally the authors gratefully acknowledge the financial support for parts of their work from the Erlangen Graduate School in Advanced Optical Technologies (SAOT) within the framework of the German Excellence Initiative by the German Research Foundation (DFG).

\section{References}

[1] Martin Krämer, E. K., Michael Wensing, 2016, "Flashboiling-induced targeting changes in gasoline direct injection sprays," international J of Engine Research, 17(1), p. 10.

[2] RD Oza, J. S., 1983, "An experimental and analytical study of flash-boiling fuel injection," SAE technical paper 830590.

[3] Min Xu, Y. Z., Wei Zeng, Gaoming Zhang and Ming Zhang, 2013, "Flash Boiling: Easy and Better Way to Generate Ideal Sprays than the High Injection Pressure," SAE Int. J. Fuels Lubr., 6(1).

[4] Andrew Wood, G. W., Jerome Helie, 2014, "Flash Boiling Sprays produced by a 6-hole GDI Injector," 17th International Symposium on Applications of Laser Techniques to Fluid MechanicsLisbon.

[5] Wei Zeng, M. X., Gaoming Zhang, Yuyin Zhang, David J. Cleary, 2012, "Atomization and vaporization for flash-boiling multi-hole sprays with alcohol fuels," Fuel, 95, p. 11.

[6] Krämer, M., 2016, Einfluss motorischer Randbedingungen auf die Spraycharakteristik bei direkteinspritzenden Ottoverfahren.

[7] Günther, A., 2016, Zerstäubung überhitzter Fluide - Einfluss der Durchflusscharakteristik auf das Spray, Friedrich-Alexander-Universität Erlangen-Nürnberg (FAU).

[8] Hung, D., Harrington, D., Gandhi, A., Markle, L. et al., 2009, "Gasoline Fuel Injector Spray Measurement and Characterization - A New SAE J2715 Recommended Practice," SAE Int. J. Fuels Lubr., 1(1), p. 14. 